\title{
Atomic structure of granulin determined from native nanocrystalline granulovirus using an X-ray free-electron laser
}

Cornelius Gati ${ }^{1}$, Dominik Oberthuer ${ }^{2}$, Oleksandr Yefanov², Richard D. Bunker ${ }^{3}$, Peter Metcalf4, Anton Barty², Henry N. Chapman ${ }^{2,5,6^{*}}$

\footnotetext{
${ }^{1}$ School of Medicine, Department of Structural Biology, Stanford University, 318 Campus Drive West, Clark Center, Room W250, Stanford, CA 94305-5151 USA

${ }^{2}$ Center for Free-Electron Laser Science, Deutsches Elektronen-Synchrotron DESY, Notkestrasse 85, 22607 Hamburg, Germany

3 Friedrich Miescher Institute for Biomedical Research, Maulbeerstrasse 66, 4058 Basel, Switzerland

${ }^{4}$ School of Biological Sciences, The University of Auckland, Private Bag 92019, Auckland Mail Centre, Auckland 1142, New Zealand

${ }^{5}$ Department of Physics, University of Hamburg, Jungiusstrasse 6, Hamburg, 20355 Germany

6 Center for Ultrafast Imaging, University of Hamburg, Luruperstrasse, Hamburg, 20355 Germany
}

*: To whom correspondence should be addressed: henry.chapman@desy.de or peter.metcalf@auckland.ac.nz

To understand how molecules function in biological systems, new methods are required to obtain atomic resolution structures from biological material under physiological conditions. Intense femtosecond-duration pulses from X-ray freeelectron lasers (XFELs) can outrun most damage processes, vastly increasing the tolerable dose before the specimen is destroyed. This in turn allows structure determination from crystals much smaller and more radiation sensitive than previously considered possible, allowing data collection from room temperature structures and avoiding structural changes due to cooling. Regardless, highresolution structures obtained from XFEL data mostly use crystals far larger than $1 \mu \mathrm{m}^{3}$ in volume, while the X-ray beam is often attenuated to protect the detector from damage caused by intense Bragg spots. Here, we describe the $2 \AA$ resolution structure of native nanocrystalline granulovirus occlusion bodies (OBs) that are less than $0.016 \mu \mathrm{m}^{3}$ in volume using the full power of the Linac Coherent Light Source (LCLS) and a dose up to 1.3 GGy per crystal. The crystalline shell of 
granulovirus OBs consists, on average, of about 9,000 unit cells, representing the smallest protein crystals to yield a high-resolution structure by X-ray crystallography to date. The XFEL structure shows little to no evidence of radiation damage and is more complete than a model determined using synchrotron data from recombinantly produced, much larger, cryo-cooled granulovirus granulin microcrystals. Our measurements suggest that it should be possible, under ideal experimental conditions, to obtain data from protein crystals with only 100 unit cells in volume using currently available XFELs and suggest that single-molecule imaging of individual biomolecules could almost be within reach. 